\title{
EFFECTS OF VEGETATION COVER/LAND USE AND SLOPE ASPECT ON SURFACE SOIL PROPERTIES NEAR THE COPPER SMELTER FACTORY IN MURGUL, TURKEY
}

\author{
KUCUK, M. ${ }^{1}-$ YENER, I. ${ }^{1 *}-$ DUMAN, A. ${ }^{2}$ \\ ${ }^{1}$ Faculty of Forestry, Artvin Coruh University, Artvin, Turkey \\ ${ }^{2}$ Artvin Vocational School, Artvin Coruh University, Artvin, Turkey \\ *Corresponding author \\ e-mail: ismetyener@hotmail.com; phone: +90-505-351-0085; fax: +90-466-215-1034
}

(Received $4^{\text {th }}$ May 2019; accepted $16^{\text {th }}$ Jul 2019)

\begin{abstract}
The mining activities, one of the most drastic examples of human intervention to nature, have considerably deteriorated the environment. Although being one of the smallest and least populated cities of Turkey, Artvin is notably affected by mining in many ways such as acid rains, water contamination and medical problems. The activities also affect soil properties. The present study aimed to assess the effects of restoration and reclamation of the former smelter factory and determine the changes in some soil properties according to land use/land cover (LULC) and slope aspect. With this purpose, Pearson correlation and two-way ANOVA methods were used. Results showed that while LULC significantly affected ( $\mathrm{p}<0.05$ ) all soil properties except silt content, bulk density, $\mathrm{CaCO}_{3}$ content, $\mathrm{C}: \mathrm{N}$ ratio, $\mathrm{Cd}$ and $\mathrm{Zn}$; slope aspect significantly affected less soil properties such as sand, silt, $\mathrm{pH}, \mathrm{EC}, \mathrm{CaCO}_{3}$ and $\mathrm{Pb}$. The interaction between factors also significantly affected $(\mathrm{p}<0.05)$ soil properties like LULC. According to partial eta squared $(\eta 2)$ values, while the most significantly affected soil properties by LULC, slope aspect and their interaction were found to be $\mathrm{Cr}(0.93)$, Clay (0.59) and $\mathrm{Cr}(0.94)$, respectively; the ones least affected were found to be total nitrogen (0.12), $\mathrm{CaCO}_{3}(0.14)$ and total nitrogen for 63-day (0.18), respectively. Despite the black alder's and black locust's lack of phytoremediation abilities concerning soil heavy metal content except $\mathrm{Pb}$, Alnus glutinosa and Robinia pseudoacacia may be suggested for the restoration and reclamation of mining soils in terms of phytoremediation beside their advantages such as improving nitrogen mineralization.
\end{abstract}

Keywords: copper mining, two-way ANOVA, black alder, black locust, partial eta squared

\section{Introduction}

The human being has been forced to find new resources for energy and row material and to change land use because of increasing population since ancient times. Pollution, deforestation, erosion, drought-desertification and global climate change are only a few negative results of human activities causing the land use and land cover changes (LUCC) (Deng et al., 2013; Mahmood et al., 2010). The mining, one of the human activities, also has some negative impacts on the environment such as hydrologic, biologic, societal, blasting, subsidence, air quality and land surface effects (Allgaier, 1997).

Artvin has recently come into question with LUCC due to the construction of hydropower plants and mining activities. In this scope, there are 72 mineral deposit reserves determined in Artvin province consisting of $44-\mathrm{Cu}-\mathrm{Pb}-\mathrm{Zn}, 1-\mathrm{Fe}, 17-\mathrm{Mn}, 5-\mathrm{Cu}-$ Mo and 5-Au (TUIK, 2013). Total copper reserve including Artvin province and Murgul is 329.681 tones (DPT, 2001). Murgul mining entity, dated back to Genoese era, operated in between 1907-1914 by Russians, reactivated in 1951 with the copper smelter by General Directorate of Mineral Research and Exploration (MTA). The 
sulphuric acid factory, operated in between 1963-1975 and 1986-199 was also added to the entity to prevent $\mathrm{SO} 2$ emission. The $\mathrm{SO} 2$ production, emitted from the factory in between 1951-197 was estimated as 795431 tons (Erdin, 1983; Oruc, 2013). Damages to the ecosystems such as soil loss, lesions and color changes especially on the leaves of fruit trees, lower $\mathrm{pH}$ and organic matter content in soil and sulphur accumulation in needles around smelter due to $\mathrm{SO} 2$ and other emissions from the factory, were reported by some researchers (Erdin, 1983; Hutchinson and Whitby, 1977; Oruc, 2013). The current physical and chemical properties of soils which influence some vital processes such as carbon storage and nitrogen retention are determined by the duration and the type of land use. On the other hand, heavy metal contamination of soil is another environmental problem, which threatens human health beside the environment by affecting the food chain (Liu et al., 2017; Yesilonis et al., 2016). The area around the factory was planted by Artvin Forest Directorate in 1996 with Robinia pseudoacacia and Alnus glutinosa sp. to remediate the area. In remediation, beside above mentioned species pine, oak, lime, larch, poplar and birch also have been used in the world. The afforestation of these sites after soil amelioration results in good results (Fischer and Fischer, 2006).

Mining activities cause extreme influences on ecosystem and soil properties and it requires more time to restore soil quality. Therefore, assessing the changes in soil properties is important to see the effects of reclamation of mining areas (Shrestha and Lal, 2011). One of the most important factors affecting restoration and quality of soil is LUCC resulted from human-being, which have an effect on the changes in biological properties. Biomass and forest floor affect many features such as microbial biomass, soil nutrients and soil organic matter (Qi et al., 2018) which have a vital role in carbon balance. The amount of carbon sequestrated in soil in global scale has been estimated 3.3 and 4.5 times more than that in atmosphere and biosphere, respectively (Lal, 2004). The soil properties are expected to change by also slope aspect which is one of the topographic features and closely related to local climate (e.g. precipitation, evaporation and solar incident radiation) (Xu et al., 2008) beside LUCC. Therefore, the slope aspect was taken as the main second factor expected to change selected soil properties.

The aim of the present study was to test our hypothesis that assumes afforestation with black alder and black locust compared to grasslands would improve the soil properties, affecting soil quality after the mining (smelter factory).

\section{Materials and methods}

\section{Site description}

The study area is located between $41^{\circ} 15^{\prime} 53^{\prime \prime}-41^{\circ} 16^{\prime} 21^{\prime \prime} \mathrm{N}$ latitudes and $41^{\circ} 33^{\prime} 21^{\prime \prime}$ - 41 ${ }^{\circ} 34^{\prime} 04$ " E longitudes (Fig. 1). It is found in Murgul district which is composing of two deep valley surrounded by high mountains with steep slopes (Acatay, 1968). The average elevation of the study site is $600 \mathrm{~m}$ above sea level. This region is characterized by mountainous topography with steep slopes. The average slope ranges between 40 and $60 \%$. Soil parent material varies widely from sedimentary marl to igneous dacite, rhyodacite, rhyolite (Anonymous, 2002). The soil texture ranges from clay loam, silty clay loam to sandy loam, loam and loamy clay. Humus form includes both moder and mulls. The soil in the district is generally acidic due to high precipitation causing leaching of base cations (Yener, 2013). 


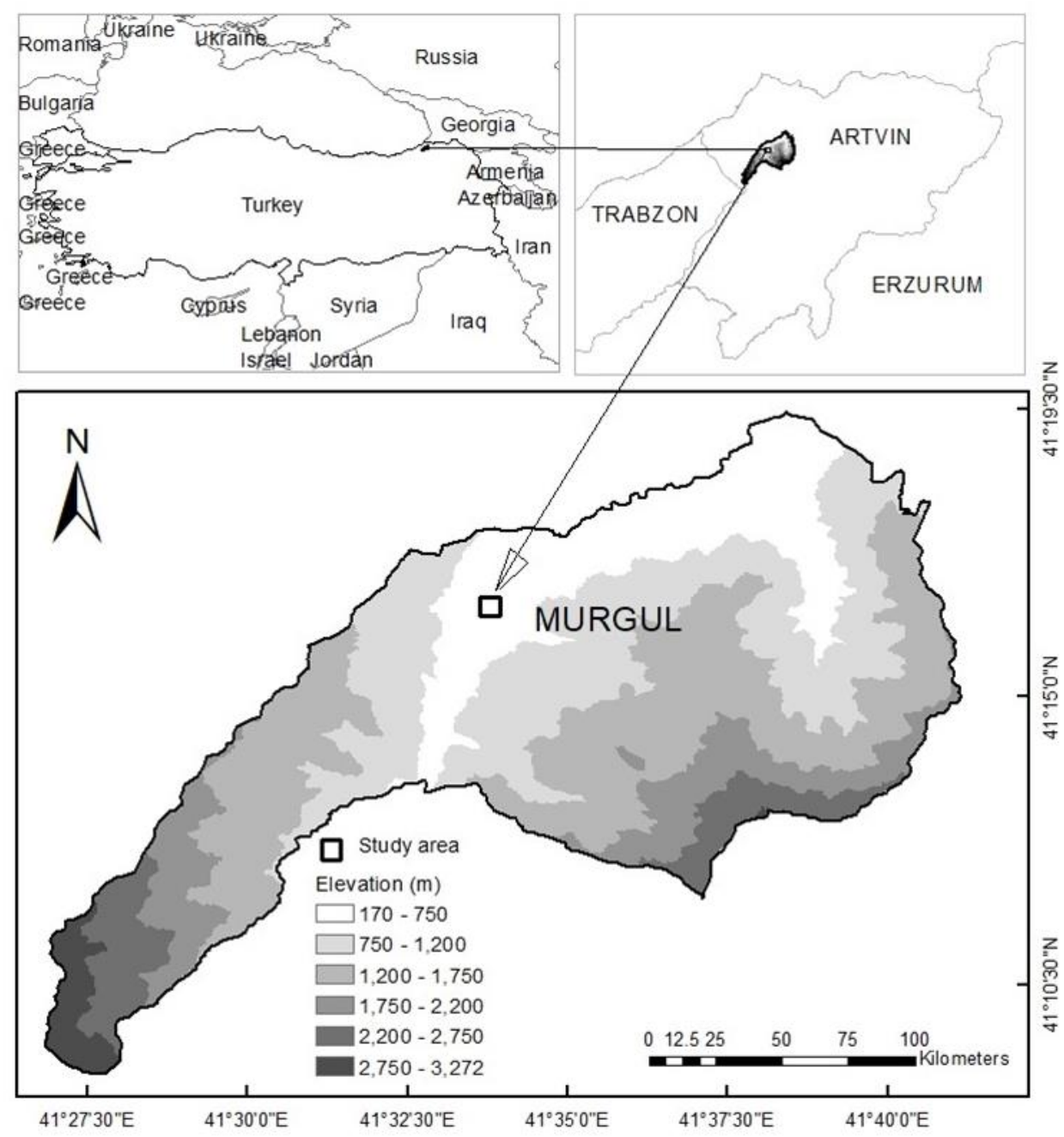

Figure 1. Location of the study area in Murgul province, northeast Turkey

The study area shows Eastern Black Sea climate under Black Sea macroclimate type, temperate in winter, warmer in summer and quite rainy in all seasons. According to the extrapolated climate data from the meteorological station closest to the study area, the annual mean temperature and the annual mean precipitation are $11.2^{\circ} \mathrm{C}$ and $1213 \mathrm{~mm}$, respectively. Mean annual minimum and maximum temperature are $7.1{ }^{\circ} \mathrm{C}$ and $16.6^{\circ} \mathrm{C}$, respectively (DMI, 2005). The growing season in the region is about six months (from May to October). The climate type is AB'1rb'3 (perhumid, mesothermal with no season of water deficiency, close to oceanic climate) according to Thornthwaite (1948).

In the district, along with oriental spruce, beech (Fagus orientalis), fir (Abies nordmanniana), Scotch pine (Pinus sylvestris) and alder (Alnus glutinosa) species are found. Regarding understory vegetation, European mountain ash (Sorbus aucuparia), Caucasina laurel (Prunus officinalis), black sea holly (Ilex colchica), common elder (Sambucus nigra), European blueberry (Vaccinium myrtillus), Caucasian honeysuckle (Lonicera caucasica), fly honeysuckle (Lonicera xylosteum), rock red currant (Ribes biebersteinii), oriental currant (Ribes orientale), pontic azalea (Rhododendron ponticum), and yellow azalea (Rhododendron luteum) species are widely spread on the forest floor (Ansin, 1980). 


\section{Soil sampling and analyses}

The sampling was done during the summer season of 2017. 54 sample plots were determined and equally distributed in 2-slope aspects such as north facing slopes (NFS) and south facing slopes (SFS), and 3 land use/land cover (LULC) types including 18black alder, 18-black locust and 18-grassland with 9-replicate $(2$ slope aspects $\times 3$ LULC $\times$ 9-replicate $=54$ ). Top soil is the most common used part in related to LUCCsoil quality studies because of its susceptibility to LUCC (Gol, 2017). Therefore, 54-top soil (disturbed) and 54-soil-core (undisturbed) samples were collected at $0-20 \mathrm{~cm}$ soil depth to determine some soil properties. All soil samples were air-dried and sieved through 2-mm mesh before analyses. All measurements were done with two repetitions. Bulk density (BD) was determined by steel core sampler after soil samples were ovendried at $105{ }^{\circ} \mathrm{C}$ (Blake and Hartge, 1986). Soil skeleton (Stoniness) was determined using undisturbed soil core samples. Soil texture analysis was done using Bouyoucos hydrometer method (Bouyoucos, 1962). Soil acidity $(\mathrm{pH})$ and electrical conductivity (EC) were determined in 1:2.5 and 1:5 soil: distilled water ratio, respectively with glass electrode, and organic carbon (SOC) was determined using Walkley \& Black wet digestion method and soil organic matter was calculated by multiplying percent of organic carbon with a factor of 1.7242001 (Kalra and Maynard 1991). Soil lime content $\left(\mathrm{CaCO}_{3}\right)$ was determined with Schreiber's Calcimeter (Nelson, 1982). Total nitrogen (TN) was determined following Kjelhdal digestion, distillation, and titration method (Pansu and Gautheyrou, 2007). Carbon-nitrogen ratio (C:N) was determined as the ratio of total carbon to total nitrogen. Potentially mineralisable-N $\left(\mathrm{NO}_{3}{ }^{-}-\mathrm{N}\right.$ plus $\left.\mathrm{NH}_{4}{ }^{+}-\mathrm{N}\right)$ was determined by microdestillation method following Bremner and Keeney (1965) in the beginning and at the $63^{\text {rd }}$ days of incubation, and the net mineral nitrogen was calculated for 63 days (Ammonium for 63 days: Ammon63; Nitrate for 63 days: Nitrate63; Total $\mathrm{N}$ for 63 days: T63) using the difference between beginning and $63^{\text {rd }}$ day (Guleryuz et al., 2007). Heavy metals in soil such as Cadmium (Cd), Chromium $(\mathrm{Cr})$, Cupper $(\mathrm{Cu})$, Lead $(\mathrm{Pb})$ and Zinc $(\mathrm{Zn})$ were determined with ICP-OES following EPA (1996) 3051 procedure.

\section{Data analyses}

Pearson correlation analysis was performed to determine the significant $(\mathrm{p}<0.005)$ relationships among variables. Then, two-way analysis of variance (ANOVA) was used following the general linear model procedure to determine the effects of factors (vegetation cover, slope aspect) and their interaction (vegetation cover*slope aspect) on dependent variables. Tukey HSD test were used to determine differences between vegetation cover groups. To interpret the interaction, six new variables were computed with original cells as a level. Then, one-way ANOVA was run and simple main effects from one-way ANOVA was examined using the contrast results 2005). An anthropogenic index (AI) which indicates antropogenic impacts on selected soil properties with a specific number, using partial eta-squared values from two-way ANOVA, developed by Zhang et al. (2012) was used using the formula below:

$$
\mathrm{AI}=\frac{1}{n}+\sum_{i=1}^{n}\left(\frac{\eta 2 \mathrm{ai}}{\eta 2 \mathrm{ni}+\eta 2 \mathrm{ai}}\right) \times 100
$$


where $\eta 2_{a i}$ and $\eta 2_{n i}$ refer to partial eta-squared values of land use and soil series for a certain soil property, respectively, and $n$ is the number of soil properties. In this study, $n=19$. Anthropogenic index ranges from 0 to 1 : the larger the AI value, the higher the anthropogenic impact. All statistical analyses were performed using SPSS v20.0 (IBM.Corp, 2011).

\section{Results and discussion}

\section{Changes in physical soil properties}

Soil texture among the land uses ranged from sandy loam to loamy clay. The texture classes were determined as loamy clay (44\%), clayey loam (44\%) and the remaining (12\%) for black locust; loamy clay (50\%), clayey loam (28\%) and the remaining (22\%) for black alder; and sandy loam (39\%), loam (33\%) and the remaining (28\%) for grasslands. Sand content of soil differed significantly $(\mathrm{p}<0.001)$ both for vegetation cover and slope aspect (Fig. 2b; Table 1). Sand content of soil on NFS (60.3\%) was found significantly $(\mathrm{p}<0.001)$ higher than that on SFS $(52.1 \%)$. Sand content of grassland soils $(64.9 \%)$ was significantly $(\mathrm{p}<0.001)$ higher than that of black alder $(52.8 \%)$ and black locust $(51.4 \%)$. Mean sand content did not differ significantly $(\mathrm{p}=0.33)$ for their interaction (Table 1$)$.

Clay content of soils also differed significantly $(\mathrm{p}<0.01)$ with vegetation cover, slope aspect and their interaction (Fig. 2a; Table 1). Clay content of NF slope soils $(17.3 \%)$ was found significantly lower than that of SF slope soils $(27.1 \%)$. Clay content of grassland soils $(14.3 \%)$ was significantly $(\mathrm{p}<0.001)$ lower than that of black alder (26.1\%) and black locust (26.3\%). Clay content of NF black alder soils (19.1\%) was found significantly $(\mathrm{p}<0.001)$ lower than that of SF ones $(33.5 \%)$ and mean clay content of NF black alder soils $(19.1 \%)$ was found significantly $(\mathrm{p}=0.015)$ higher than that of NF grassland soils (12.0\%) (Table 1). The lower clay and higher sand contents in grasslands, having little or no protective vegetation cover, may be due to the removal of clay by erosion (Miheretu and Yimer, 2018; Tsehaye and Mohammed, 2013; Bewket and Stroosnijder, 2003). Hung et al. (2017) stated that the higher clay content in tree systems (e.g. forests) compared to other land uses may attribute to the protective cover by tree crowns, roots and litter reducing soil erosion. BD in the present study ranged from $1.38 \mathrm{gr} / \mathrm{cm} 3$ to $1.84 \mathrm{gr} / \mathrm{cm} 3$ and was not significantly affected by any of the factors according to two-way ANOVA ( $\mathrm{p}>0.05)$ (Table 1). BD in forests was found lower than that in other land uses (e.g. grassland) by some other researchers (Assefa et al., 2017; Gol, 2017; Tesfaye et al., 2016; Toohey et al., 2018; Yesilonis et al., 2016). The lower BD in forests as compared to the other land uses can be attributed to higher clay content and loosening of the surface soil with plant root growth (Qi et al., 2018). Vegetation cover, slope aspect and their interaction significantly affected soil stoniness $(p<0.001)$. The stoniness of soil was not differed significantly between NFS and SFS $(\mathrm{p}=0.91)$. The stoniness of grassland soil $(46.9 \%)$ was higher than that of black alder $(38.9 \%)$ and black locust $(35.6 \%)$ soils $(\mathrm{p}<0.005)$. The interaction between vegetation cover and slope aspect also significantly affected soil stoniness (Fig. 2c; Table 1). Soil stoniness of SF grassland $(53.8 \%)$ was significantly higher than that of black alder $(32.2 \%)$ and black locust $(32.0 \%)(\mathrm{p}<0.001)$. The slope aspect significantly affected only grassland soil $(\mathrm{p}=0.005)$. The soil stoniness of NF grassland $(35.8 \%)$ was found lower than that of SF grassland (53.8\%). 
Table 1. Physical properties (mean \pm standard error) of surface soils according to LULC and slope aspect

\begin{tabular}{|c|c|c|c|c|c|c|}
\hline LULC & SASP & $\begin{array}{c}\text { Sand } \\
(\%)\end{array}$ & $\begin{array}{c}\text { Clay } \\
(\%)\end{array}$ & $\begin{array}{l}\text { Silt } \\
(\%)\end{array}$ & $\begin{array}{c}\text { BD } \\
\left(\mathrm{gr} / \mathrm{cm}^{3}\right)\end{array}$ & $\begin{array}{l}\text { Stoniness } \\
(\%)\end{array}$ \\
\hline \multirow{2}{*}{ Black locust } & NFS & $55.9 \pm 0.9$ _a_A & $19.1 \pm 0.8$ _a_A & $25.0 \pm 0.7$ a_A & $1.6 \pm 0.0 \_\mathrm{a} \_\mathrm{A}$ & $39.2 \pm 1.5 \_\mathrm{a} \_\mathrm{A}$ \\
\hline & SFS & 46.9 1.9 a_A & $33.5 \pm 1.1 \_$b_A & $19.6 \pm 1.4$ _a_A & $1.6 \pm 0.0 \_\mathrm{a} \_\mathrm{A}$ & $32.0 \pm 1.6$ a_A \\
\hline \multirow{2}{*}{ Black alder } & NFS & $58.4 \pm 3.2 \_\mathrm{a} \_\mathrm{AB}$ & $20.7 \pm 2.1$ _a_A & $20.9 \pm 1.4$ _a_A & $1.7 \pm 0.0 \_$a_A & 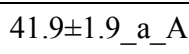 \\
\hline & SFS & $47.2 \pm 2.5$ b_A & $31.4 \pm 2.0 \_$b_A & $21.4 \pm 0.9$ _a_A & $1.5 \pm 0.0 \_\mathrm{a} \_\mathrm{A}$ & $35.8 \pm 3.8$ _a_A \\
\hline \multirow{2}{*}{ Grassland } & NFS & $66.6 \pm 2.4$ _a_B & $12.0 \pm 1.3$ _a_B & $21.4 \pm 2.6 \_$a_A & $1.6 \pm 0.0 \_\mathrm{a} \_\mathrm{A}$ & 39.9 3.4 _a_A \\
\hline & SFS & $62.0 \pm 1.8$ _a_B & $16.6 \pm 1.0 \_\mathrm{a} \_\mathrm{B}$ & $21.4 \pm 1.7$ _a_A & $1.6 \pm 0.0 \_\mathrm{a} \_\mathrm{A}$ & $53.8 \pm 2.2 \_$b_B \\
\hline \multirow{3}{*}{$\begin{array}{l}\text { Two-way } \\
\text { ANOVA }\end{array}$} & LULC & $\begin{array}{c}\mathrm{F}=20.1 \\
\mathrm{p}<0.001\end{array}$ & $\begin{array}{c}\mathrm{F}=43.8 \\
\mathrm{p}<0.001\end{array}$ & $\begin{array}{c}\mathrm{F}=0.0 \\
\mathrm{p}>0.05\end{array}$ & $\begin{array}{c}\mathrm{F}=0.0 \\
\mathrm{p}>0.05\end{array}$ & $\begin{array}{c}\mathrm{F}=10.1 \\
\mathrm{p}<0.001\end{array}$ \\
\hline & SASP & $\begin{array}{l}\mathrm{F}=20.3 \\
\mathrm{p}<0.001\end{array}$ & $\begin{array}{l}\mathrm{F}=68.5 \\
\mathrm{p}<0.001\end{array}$ & $\begin{array}{l}F=1.7 \\
p>0.05\end{array}$ & $\begin{array}{l}F=0.0 \\
p>0.05\end{array}$ & $\begin{array}{c}\mathrm{F}=0.0 \\
\mathrm{p}<0.001\end{array}$ \\
\hline & Interaction & $\begin{array}{c}\mathrm{F}=1.1 \\
\mathrm{p}>0.05\end{array}$ & $\begin{array}{l}\mathrm{F}=5.7 \\
\mathrm{p}<0.01\end{array}$ & $\begin{array}{l}\mathrm{F}=2.2 \\
\mathrm{p}>0.05\end{array}$ & $\begin{array}{l}\mathrm{F}=3.1 \\
\mathrm{p}>0.05\end{array}$ & $\begin{array}{l}\mathrm{F}=10.6 \\
\mathrm{p}<0.001\end{array}$ \\
\hline
\end{tabular}

LULC: land-use/land cover, SASP: slope aspect, NFS: north facing slopes, SFS: south facing slopes, BD: bulk density. By slope aspect, values of same LULC follow the various lower-case letters indicate significant difference. By LULC, values of same slope aspect follow the various upper-case letters differ significantly

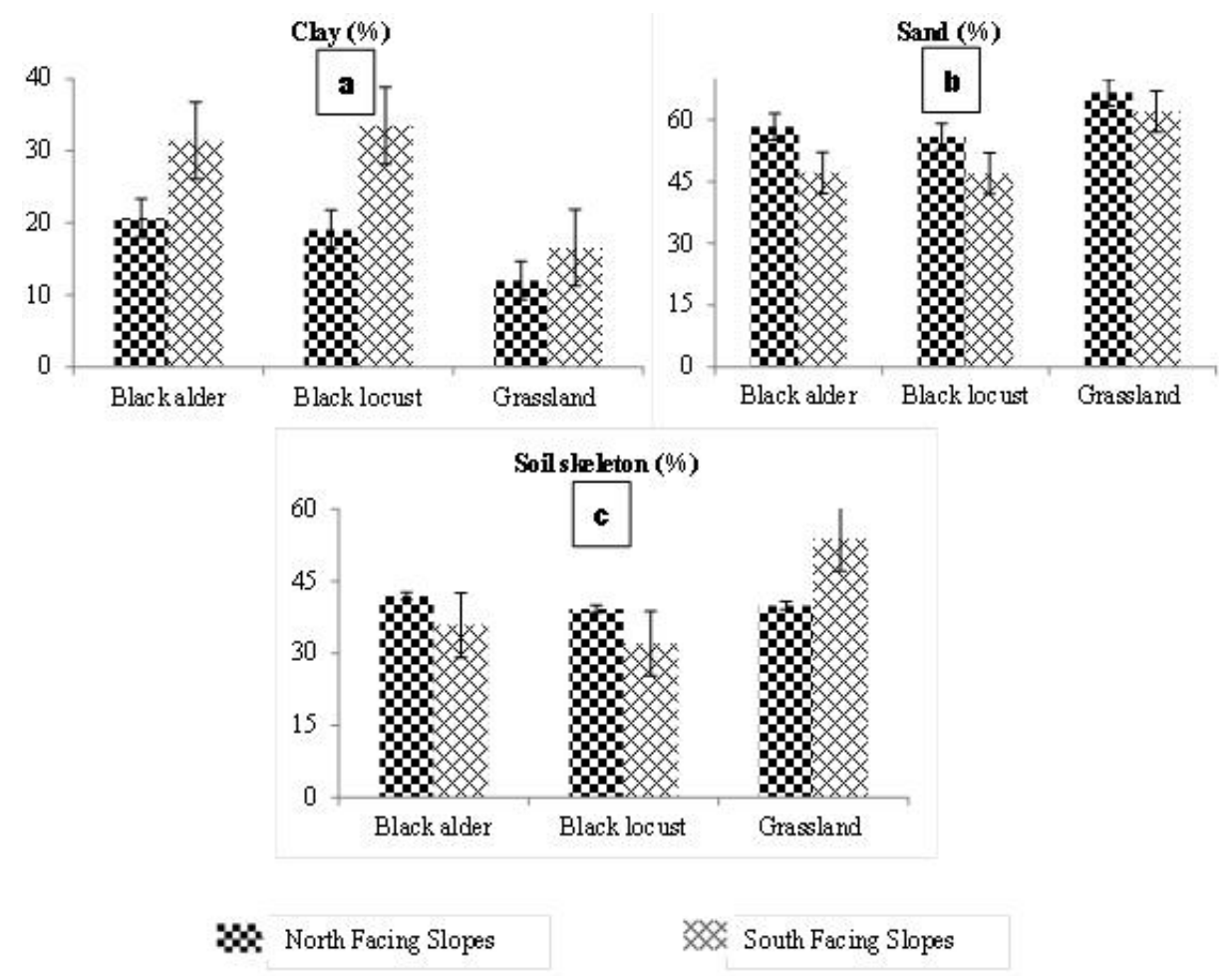

Figure 2. Physical soil properties significantly affected by LULC and slope aspect

\section{Changes in chemical soil properties}

The $\mathrm{pH}$ in the study area ranged from 3.93 (very strongly acidic) to 7.79 (weakly alkaline) according to Hazelton and Murphy (2016). This range is consistent with other 
studies (Kuo et al., 1983). $\mathrm{pH}$ was significantly affected by vegetation cover $(\mathrm{p}<0.001)$, slope aspect $(\mathrm{p}=0.005)$ and their interaction $(\mathrm{p}<0.001)($ Fig. 3a; Table 3). $\mathrm{pH}$ of grassland soils (5.29) was found significantly lower than that of black locust (6.03) and black alder (6.72) soils ( $p<0.001)$. As the slope aspect changes from north to south for all vegetation covers, soil $\mathrm{pH}$ significantly increased from 5.82 to 6.21 (Table 3). The interaction also affected soil $\mathrm{pH}(\mathrm{p}<0.001)$. While, black locust soil $\mathrm{pH}$ was significantly found lower than that of other vegetation cover soils in NFS, grassland soil $\mathrm{pH}$ was significantly lower than that of other vegetation cover soils in SFS. The change of land use from forests to grasslands resulted in a decrease in soil $\mathrm{pH}$ may be attributed to either removal of basic cations by harvesting/grazing or leaching of them because of sparse vegetation cover 2018). Shrestha and Lal (2011) also reported a significant increase in soil pH after reclamation of soil. Some researchers (Kucuk, 2013; Tufekcioglu and Kucuk, 2004) reported the results unlike ours. Kucuk (2013) for example, reported that soil $\mathrm{pH}$ in forests (5.35) was lower than that in grasslands (6.87).

Soil EC in the study area changed between 19.5 and $421 \mu \mathrm{S} / \mathrm{cm}$ which is in the class of non-saline soils meaning salinity effects are mostly negligible according to Hazelton and Murphy (2016). All factors including also interaction significantly affected soil EC ( $\mathrm{p}<0.001$ ) (Fig.3b; Table 3). EC significantly decreased in the order of black alder $>$ black locust $>$ grasslands. As the aspect changed from north to south, EC increased from 97.5 to $179.1 \mu \mathrm{S} / \mathrm{cm}$. According to interaction between two-factors, while the lowest soil EC in north and south aspects was determined in black locust and grasslands respectively as 40.1 and $29.9 \mu \mathrm{S} / \mathrm{cm}$ (Table 3). This change may be attributed to the leaching of soil bases and subsequent reduction in soil carbonate (Biro et al., 2013). The correlation between $\mathrm{EC}$ and $\mathrm{CaCO}_{3}$ in the study area was significant $(\mathrm{r}=0.71$ and $\mathrm{p}<0.01)($ Table 2$)$.

Table 2. Pearson correlation coefficient ( $r$ ) between selected soil properties

\begin{tabular}{|c|c|c|c|c|c|c|c|c|c|c|c|c|c|c|c|c|c|c|c|c|}
\hline & & (1) & (2) & (3) & (4) & (5) & (6) & (7) & (8) & (9) & (10) & (11) & (12) & (13) & (14) & (15) & (16) & (17) & (18) & (19) \\
\hline (1) & Sand & 1 & & & & & & & & & & & & & & & & & & \\
\hline (2) & Clay & $-.87^{* *}$ & 1 & & & & & & & & & & & & & & & & & \\
\hline (3) & Silt & $-.41^{* *}$ & -.09 & 1 & & & & & & & & & & & & & & & & \\
\hline (4) & $\mathrm{pH}$ & $-.35^{* *}$ & $.53^{* *}$ & $-.28^{*}$ & 1 & & & & & & & & & & & & & & & \\
\hline (5) & $\mathrm{OM}$ & $.48^{* *}$ & $-.48^{* *}$ & -.08 & $-.38^{* *}$ & 1 & & & & & & & & & & & & & & \\
\hline (6) & $\mathrm{CaCO} 3$ & $-.38^{* *}$ & $.47^{* * *}$ & -.10 & $.53^{* *}$ & -.20 & 1 & & & & & & & & & & & & & \\
\hline (7) & $\mathrm{EC}$ & $-.50^{* *}$ & $.69^{* *}$ & $-.27^{*}$ & $.85^{* *}$ & $-.28^{*}$ & $.71^{* *}$ & 1 & & & & & & & & & & & & \\
\hline (8) & $\mathrm{TN}$ & -.03 & -.02 & .10 & -.07 & $.35^{* *}$ & -.13 & -.01 & 1 & & & & & & & & & & & \\
\hline (9) & $\mathrm{CN}$ & $.33^{*}$ & $-.33^{*}$ & -.06 & -.16 & $.27^{*}$ & -.12 & -.21 & $-.49^{* *}$ & 1 & & & & & & & & & & \\
\hline (10) & $\mathrm{BD}$ & .01 & -.04 & .04 & .03 & -.12 & -.10 & -.08 & .06 & -.13 & 1 & & & & & & & & & \\
\hline (11) & Stoniness & $.36^{* *}$ & $-.38^{* *}$ & -.03 & $-.41^{* *}$ & $.51^{* *}$ & -.07 & $-.34^{*}$ & $.36^{* *}$ & -.04 & .12 & 1 & & & & & & & & \\
\hline (12) & Ammon63 & $.45^{* *}$ & $-.51^{* *}$ & .03 & $-.51^{* *}$ & $.36^{* *}$ & -.09 & $-.41^{* *}$ & -.12 & .13 & .12 & $.55^{* *}$ & 1 & & & & & & & \\
\hline (13) & Nitrat63 & -.04 & .17 & -.24 & $.43^{* *}$ & -.08 & -.05 & $.28^{*}$ & .24 & -.09 & .05 & -.17 & $-.42^{* *}$ & 1 & & & & & & \\
\hline (14) & T63 & .22 & -.12 & -.24 & .16 & .13 & -.11 & .06 & .19 & -.02 & .12 & .14 & .14 & $.84^{* *}$ & 1 & & & & & \\
\hline (15) & $\mathrm{Cd}$ & .11 & -.08 & -.07 & .02 & .07 & -.04 & .18 & .01 & -.06 & -.17 & -.10 & .02 & .28 & .30 & 1 & & & & \\
\hline (16) & $\mathrm{Cr}$ & $-.49^{* *}$ & $.57^{* *}$ & -.04 & $.69^{* *}$ & $-.51^{* *}$ & $.42^{*}$ & $.66^{* *}$ & -.30 & -.04 & -.14 & $-.71^{* *}$ & $-.49^{* *}$ & .17 & -.12 & -.07 & 1 & & & \\
\hline (17) & $\mathrm{Cu}$ & -.02 & -.15 & .32 & -.26 & -.05 & -.13 & -.16 & .05 & -.09 & $-.35^{*}$ & -.18 & .00 & -.12 & -.13 & $.38^{*}$ & .13 & 1 & & \\
\hline (18) & $\mathrm{Pb}$ & .24 & -.27 & .00 & $-.59^{* *}$ & .28 & -.27 & $-.37^{*}$ & .11 & -.03 & -.12 & $.41^{*}$ & $.53^{* *}$ & -.16 & .17 & $.45^{* *}$ & $-.67^{* *}$ & .27 & 1 & \\
\hline (19) & $\mathrm{Zn}$ & .24 & -.26 & -.02 & -.25 & .21 & -.22 & -.13 & .05 & .11 & -.20 & .00 & .14 & .23 & .33 & $.81^{* * *}$ & $-.41^{*}$ & .29 & $.66^{* * *}$ & 1 \\
\hline
\end{tabular}

**Correlation is significant at the 0.01 level. *Correlation is significant at the 0.05 level. pH: soil acidity, EC: electrical conductivity, TN: total nitrogen, CN: carbon: nitrogen ratio, BD: bulk density, stoniness: soil skeleton, Ammon6 Nitrat63 and T 63: ammonium, nitrate and total nitrogen mineralization for 63-day, $\mathrm{Cd}$ : cadmium, $\mathrm{Cr}$ : chromium, $\mathrm{Cu}$ : copper, $\mathrm{Pb}$ : lead, $\mathrm{Zn}$ : zirconium 
Table 3. Chemical properties (mean \pm standard error) of surface soils according to LULC and slope aspect

\begin{tabular}{|c|c|c|c|c|c|c|}
\hline LULC & SASP & pH & OM $(\%)$ & $\operatorname{CaCO3}(\%)$ & $\mathrm{EC}(\mu \mathrm{S} / \mathrm{cm})$ & TN (\%) \\
\hline \multirow{2}{*}{ Black locust } & NFS & $4.7 \pm 0.0 \_$a_A & $1.9 \pm 0.2 \_\mathrm{a} \_\mathrm{A}$ & $1.2 \pm 0.1$ a_A & 40.1 \pm 2.8 a_A & $0.094 \pm 0.003 \_$a_AB \\
\hline & SFS & $7.4 \pm 0.2$ b_A & $1.5 \pm 0.2 \_\mathrm{a} \_\mathrm{A}$ & 3.0 0.7 -a_A & $248.5 \pm 30.1 \_$b_A & $0.080 \pm 0.004 \_\mathrm{a} \_\mathrm{A}$ \\
\hline \multirow{2}{*}{ Black alder } & NFS & $6.4 \pm 0.1 \_\mathrm{a} \_\mathrm{B}$ & $2.7 \pm 0.3 \_\mathrm{a} \_\mathrm{A}$ & $1.6 \pm 0.3 \_\mathrm{a} \_\mathrm{A}$ & $162.6 \pm 26.5$ _a_B & $0.117 \pm 0.008$ a_A \\
\hline & SFS & $7.0 \pm 0.3$ a_A & $1.5 \pm 0.2 \_\mathrm{b} \_\mathrm{A}$ & $2.8 \pm 0.7$ a_A & 258.8 \pm 30.8 _b_A & $0.085 \pm 0.004$ b_A \\
\hline \multirow{2}{*}{ Grassland } & NFS & $6.3 \pm 0.2$ _a_B & $2.0 \pm 0.1 \_\mathrm{a} \_\mathrm{A}$ & $1.2 \pm 0.2 \_\mathrm{a} \_\mathrm{A}$ & $90.0 \pm 11.7$ a_A & $0.078 \pm 0.009$ a_B \\
\hline & SFS & 4.3 $\pm 0.1 \_b \_B$ & 3.1 $\pm 0.4 \_b \_B$ & $1.2 \pm 0.1 \_\mathrm{a} \_\mathrm{A}$ & $29.9 \pm 2.9$ _a_B & $0.096 \pm 0.007$ _a_A \\
\hline \multirow{6}{*}{$\begin{array}{l}\text { Two-way } \\
\text { ANOVA }\end{array}$} & LULC & $\mathrm{F}=38.5$ & $\mathrm{~F}=6.5$ & $\mathrm{~F}=3.2$ & $\mathrm{~F}=25.3$ & $\mathrm{~F}=3.3$ \\
\hline & LULC & $\mathrm{p}<0.001$ & $\mathrm{p}<0.01$ & $\mathrm{p}>0.05$ & $\mathrm{p}<0.001$ & $\mathrm{p}<0.01$ \\
\hline & & $\mathrm{F}=8.5$ & $\mathrm{~F}=0.0$ & $\mathrm{~F}=7.7$ & $\mathrm{~F}=22.0$ & $\mathrm{~F}=3.3$ \\
\hline & SASP & $\mathrm{p}<0.01$ & $\mathrm{p}>0.05$ & $\mathrm{p}<0.01$ & $\mathrm{p}<0.001$ & $\mathrm{p}<0.01$ \\
\hline & Interaction & $\mathrm{F}=103.0$ & $\mathrm{~F}=10.6$ & $\mathrm{~F}=1.9$ & $\mathrm{~F}=20.1$ & $F=7.6$ \\
\hline & Interaction & $\mathrm{p}<0.001$ & $\mathrm{p}<0.001$ & $\mathrm{p}>0.05$ & $\mathrm{p}<0.001$ & $\mathrm{p}<0.01$ \\
\hline LULC & SASP & $\mathbf{C N}$ & $\begin{array}{c}\text { Ammon63 } \\
\text { (kg/ha) }\end{array}$ & $\begin{array}{c}\text { Nitrat63 } \\
\text { (kg/ha) }\end{array}$ & T63 (kg/ha) & \\
\hline \multirow{2}{*}{ Black locust } & NFS & $11.6 \pm 1.1$ a_A & $28.5 \pm 1.3 \_\mathrm{a} \_\mathrm{AB}$ & 32.0 \pm 2.9 _a_A & $60.5 \pm 2.3$ _a_A & \\
\hline & SFS & $11.1 \pm 1.4$ _a_A & 23.8 \pm 3.6 _a_A & $42.5 \pm 2.8$ a_AB & $66.3 \pm 4.4$ _a_A & \\
\hline \multirow{2}{*}{ Black alder } & NFS & $13.2 \pm 1.2$ _a_A & $22.3 \pm 1.9$-a_A & 66.4 \pm 5.9 _a_B & $88.7 \pm 5.8$ _a_B & \\
\hline & SFS & $10.0 \pm 1.4$ _a_A & $21.2 \pm 1.9$ _a_A & $51.4 \pm 4.7$ a_B & 72.6 5 5.4_a_A & \\
\hline \multirow{2}{*}{ Grassland } & NFS & $25.7 \pm 11.9$-a_A & $31.2 \pm 2.2 \_\mathrm{a} \_\mathrm{B}$ & 39.7 \pm 5.0 a_A & $70.9 \pm 5.5$ _a_A & \\
\hline & SFS & $20.0 \pm 2.9$ _a_B & $40.6 \pm 2.1 \_\mathrm{a} \_\mathrm{B}$ & $30.5 \pm 2.7 \_\mathrm{a} \_\mathrm{A}$ & $71.2 \pm 3.2 \_\mathrm{a} \_\mathrm{A}$ & \\
\hline \multirow{3}{*}{$\begin{array}{l}\text { Two-way } \\
\text { ANOVA }\end{array}$} & LULC & $\begin{array}{c}F=3.3 \\
p>0.05\end{array}$ & $\begin{array}{c}\mathrm{F}=20.3 \\
\mathrm{p}<0.001\end{array}$ & $\begin{array}{c}\mathrm{F}=19.7 \\
\mathrm{p}<0.001\end{array}$ & $\begin{array}{c}\mathrm{F}=19.7 \\
\mathrm{p}<0.001\end{array}$ & \\
\hline & SASP & $\begin{array}{l}\mathrm{F}=0.0 \\
\mathrm{p}>0.05\end{array}$ & $\begin{array}{l}\mathrm{F}=0.0 \\
\mathrm{p}>0.05\end{array}$ & $\begin{array}{c}\mathrm{F}=1.8 \\
\mathrm{p}>0.05\end{array}$ & $\begin{array}{c}\mathrm{F}=1.8 \\
\mathrm{p}>0.05\end{array}$ & \\
\hline & Interaction & $\begin{array}{l}F=0.0 \\
p>0.05\end{array}$ & $\begin{array}{l}F=5.2 \\
p<0.01\end{array}$ & $\begin{array}{l}F=5.1 \\
p<0.01\end{array}$ & $\begin{array}{l}F=5.1 \\
p<0.01\end{array}$ & \\
\hline
\end{tabular}

LULC: land-use/land cover, SASP: slope aspect, NFS: north facing slopes, SFS: south facing slopes, OM: organic matter, EC: electrical conductivity, TN: total nitrogen, CN: carbon: nitrogen ratio, Ammon6 Nitrat63 and T63: ammonium, nitrate and total nitrogen mineralization for 63-day. By slope aspect, values of same LULC follow the various lower-case letters indicate significant difference. By LULC, values of same slope aspect follow the various upper-case letters differ significantly

Organic matter classes of soils in the study area ranged from weak (1.69\%) in black locust to moderate (2.59\%) in grasslands according to 2016). Organic matter content of black alder soils $(1.70 \%)$ were significantly found lower than that of grassland soils $(2.59 \%)(\mathrm{p}=0.002)$ (Fig. 3c; Table 3). Similar results were found by some other researchers (Pu et al., 2018; Wei et al., 2011; Yesilonis et al., 2016). The slope aspect alone did not affect soil organic matter $(\mathrm{p}=0.46)$. In SFS, soil organic matter content of grasslands $(3.13 \%)$ was higher than that of black locust $(1.51 \%)$ and black alder (1.49\%) (Fig. 3c; Table 3). While soil organic matter content of black alder increased from south $(1.49 \%)$ to north $(2.66 \%)$ facing slopes $(\mathrm{p}=0.02)$, that of grasslands decreased $(p=0.037)$. The positive effect of north aspect on organic matter content by also found by Qin et al. (2017). Increasing organic matter content in north aspect can be attributed to more organic carbon and more total nitrogen accumulation in the soil because of higher soil water content and less evaporation (Johnson et al., 2011). 

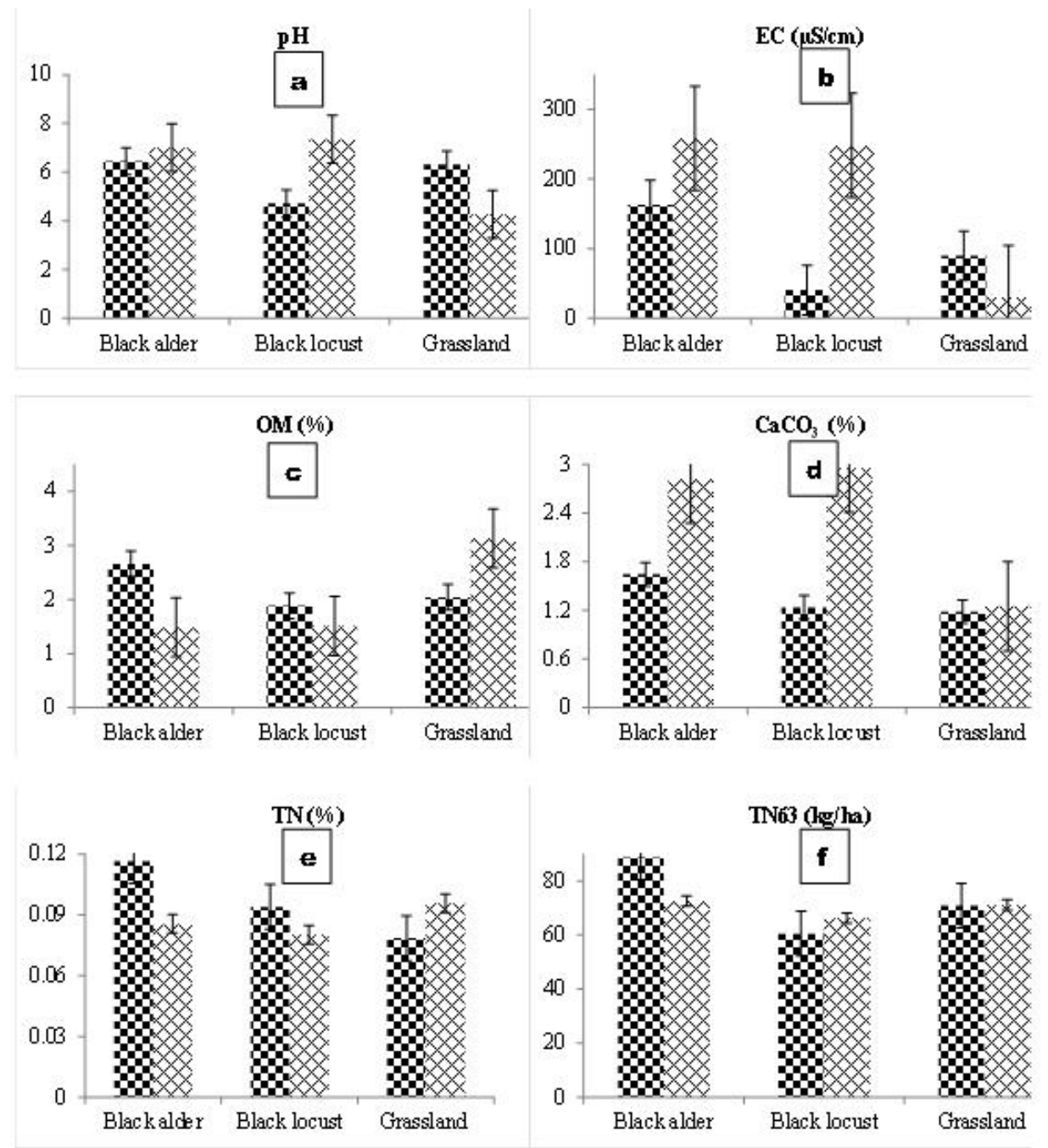

W. North Facing Slopes

Xy: South Facing Slopes

Figure 3. Chemical soil properties significantly affected by LULC and slope aspect

On the one hand, $\mathrm{CaCO}_{3}$ content of soils was not significantly affected by vegetation cover $(p=0.05)$ and vegetation cover*slope aspect interaction $(p=0.164)$, on the other hand, the content decreased from south $(2.34 \%)$ to north $(1.35 \%)$ without considering vegetation cover effect $(\mathrm{p}=0.008)$ (Fig. 3d; Table 3).

When not considered the effects of vegetation cover, soil EC in SFS $(179.1 \mu \mathrm{S} / \mathrm{cm})$ was also significantly greater than that in NFS $(97.5 \mu \mathrm{S} / \mathrm{cm})(\mathrm{p}<0.001)$. Soil EC in grasslands $(59.9 \mu \mathrm{S} / \mathrm{cm})$ was found significantly lower than that in black locust $(144.3 \mu \mathrm{S} / \mathrm{cm}, \mathrm{p}=0.01)$ and black alder $(210.7 \mu \mathrm{S} / \mathrm{cm}, \mathrm{p}<0.001)$ without considering slope aspect effect. In SFS, soil EC of grassland $(29.9 \mu \mathrm{S} / \mathrm{cm})$ was found significantly less than that of other vegetation covers $(\mathrm{p}<0.001)$. Soil EC of vegetation covers except grasslands increased from NFS to SFS (Table 3).

$\mathrm{TN}$ of soils was not affected by vegetation covers $(\mathrm{p}=0.05)$ and slope aspect $(\mathrm{p}=0.07)$ but significantly affected by their interaction. It significantly increased from south $(0.085 \%)$ to north $(0.117 \%)$ for only black alder $(\mathrm{p}=0.012)($ Fig. 3e; Table 3). That increase may be attributed to improving ecological condition depending on more 
moisture in north. Similar results were found by some other researchers (Bangroo et al., 2017; Gol, 2017; Rezaei et al., 2006). Soil TN of north -facing black alder $(0.117 \%)$ was found significantly greater than that of NF grasslands $(0.078 \%)(p=0.001)$. This can be attributed to removal of organic matter with grazing and higher level of $\mathrm{N}$ fixation in black alder (Ripley et al., 2010; Wilson et al., 2011). Similar results were reported by some other researchers (Beheshti et al., 2012; Miheretu and Yimer, 2018; Tesfaye et al., 2016; Wilson et al., 2011). Soil C:N ratio was not significantly affected by vegetation cover $(\mathrm{p}=0.05)$, slope aspect $(\mathrm{p}=0.46)$ and their interaction $(\mathrm{p}=0.87)$ (Table 3). While Miheretu and Yimer (2018) and Chacon et al. (2009) also found similar results like in the present study. Significant effects of land use on soil C:N ratio also found in some other studies (Assefa et al., 2017; Kucuk, 2013).

Looked at variables regarding 63-day nitrogen mineralization (ammonium, nitrate and total); slope aspect alone had no significant effect on 63-day nitrogen mineralization. Vegetation cover alone had significant effect on 63-day ammonium, nitrate and total nitrogen mineralization. 63-day ammonium mineralization of grassland soils $(35.9 \mathrm{~kg} / \mathrm{ha})$ was found significantly higher than that of black locust $(26.1 \mathrm{~kg} / \mathrm{ha})$ and black alder $(21.8 \mathrm{~kg} / \mathrm{ha})(\mathrm{p}<0.001)$. Higher ammonium mineralization in grassland soils can be attributed to lower $\mathrm{pH}$ (Xue et al., 2013). The correlation between NH4-N and $\mathrm{pH}$ was negative $(\mathrm{p}<0.0 \mathrm{r}=-0.59)($ Table 2). Similar results were found by Wei et al. (2011) in loess plateau of China who reported higher nitrogen mineralization in grassland soils compared to others due to lower bulk density. Nitrate and total nitrogen mineralization in 63-day period of black alder soils $(58.8 \mathrm{~kg} / \mathrm{ha}$ and $80.6 \mathrm{~kg} / \mathrm{ha})$ were found significantly higher than that of the other vegetation covers (Fig. 3f; Table 3). Higher nitrate mineralization in forest (alder) soils compared to the other land uses can be attributed to that on the one hand, black alder, being one of the $\mathrm{N}$-fixing trees provides resources both easily accessible and abundant for soil microbes and facilitates $\mathrm{N}$ mineralization, on the other hand, it contributes to the supply of organic substrates which are easily decomposed and decrease $\mathrm{C}: \mathrm{N}$ ratio (Cui et al., 2018). The higher nitrate mineralization can also be attributed to positive correlation with $\mathrm{pH}(\mathrm{r}=0.4$ $\mathrm{p}<0.01$ ) (Table 2). Similar results were found also by some other researchers (Hart et al., 1997; Tecimen et al., 2013; Uri et al., 2008). Uri et al. (2008), for example, reported higher annual net nitrogen mineralization $(99 \mathrm{~kg} / \mathrm{ha}$ ) in the birch stand than that $(51 \mathrm{~kg} / \mathrm{ha})$ of in grasslands in Estonia. Tecimen et al. (2013) explained the difference with the effect of increasing organic carbon. The 63-day ammonium mineralization of SF grassland soils $(40.6 \mathrm{~kg} / \mathrm{ha})$ was found significantly higher than that of SF black locust $(23.7 \mathrm{~kg} / \mathrm{ha})$ and black alder $(21.2 \mathrm{~kg} / \mathrm{ha})$ soils $(\mathrm{p}<0.001)$. Both in NFS and SFS, 63-day nitrate mineralization of black alder soils $(66.4 \mathrm{~kg} / \mathrm{ha}$ and $51.4 \mathrm{~kg} / \mathrm{ha}$ respectively) was significantly higher than that of grassland soils $(39.7 \mathrm{~kg} / \mathrm{ha}$ and $30.5 \mathrm{~kg} / \mathrm{ha}$ respectively). 63-day total nitrogen mineralization of NF black locust soils $(60.5 \mathrm{~kg} / \mathrm{ha})$ was lower than that of black alder soils in the same aspect $(88.7 \mathrm{~kg} / \mathrm{ha})$.

\section{Changes in heavy metal concentrations}

Average $\mathrm{Cd}$ and $\mathrm{Zn}$ contents of soils in the study area were $0.7 \mathrm{ppm}$ ( 0 to $6.3 \mathrm{ppm}$ ) and $148.5 \mathrm{ppm}$ (72 to $312 \mathrm{ppm}$ ), respectively. These values are slightly higher than the average concentrations ( $0.5 \mathrm{ppm}$ for $\mathrm{Cd}$ and $64 \mathrm{ppm}$ for $\mathrm{Zn}$ ) reported by Kabata-Pendias and Mukherjee (2007). This can be due to the copper mine near the study area. Cd and $\mathrm{Zn}$ concentrations among heavy metals did not significantly differed according to vegetation cover, slope aspect and their interaction ( $\mathrm{p}>0.05)($ Table 4). 
Cr concentration in soil ranged from 5.2 to $29.5 \mathrm{ppm}$ with the average value of $20.2 \mathrm{ppm}$. This value is consistent with the range of world mean (54 ppm) reported by Kabata-Pendias and Mukherjee (2007). Soil Cr concentration of grasslands (13.8 ppm) was found significantly lower than that of black alder $(23.3 \mathrm{ppm})$ and black locust $(24.4 \mathrm{ppm})(\mathrm{p}<0.001)$. The soil Cr content of black alder and black locust significantly increased from NFS to SFS unlike grasslands $(\mathrm{p}<0.05)$. The soil Cr content of SF grassland $(6.7 \mathrm{ppm})$ was significantly lower than that of black alder $(28 \mathrm{ppm})$ and black locust $(26.8 \mathrm{ppm})$ in the same aspect (Fig. 4a; Table 4). Higher $\mathrm{Cr}$ contents of forest soil may be related to higher $\mathrm{pH}(\mathrm{r}=0.6 \mathrm{p}<0.01)$ (Table 2) (Kabata-Pendias and Mukherjee, 2007).
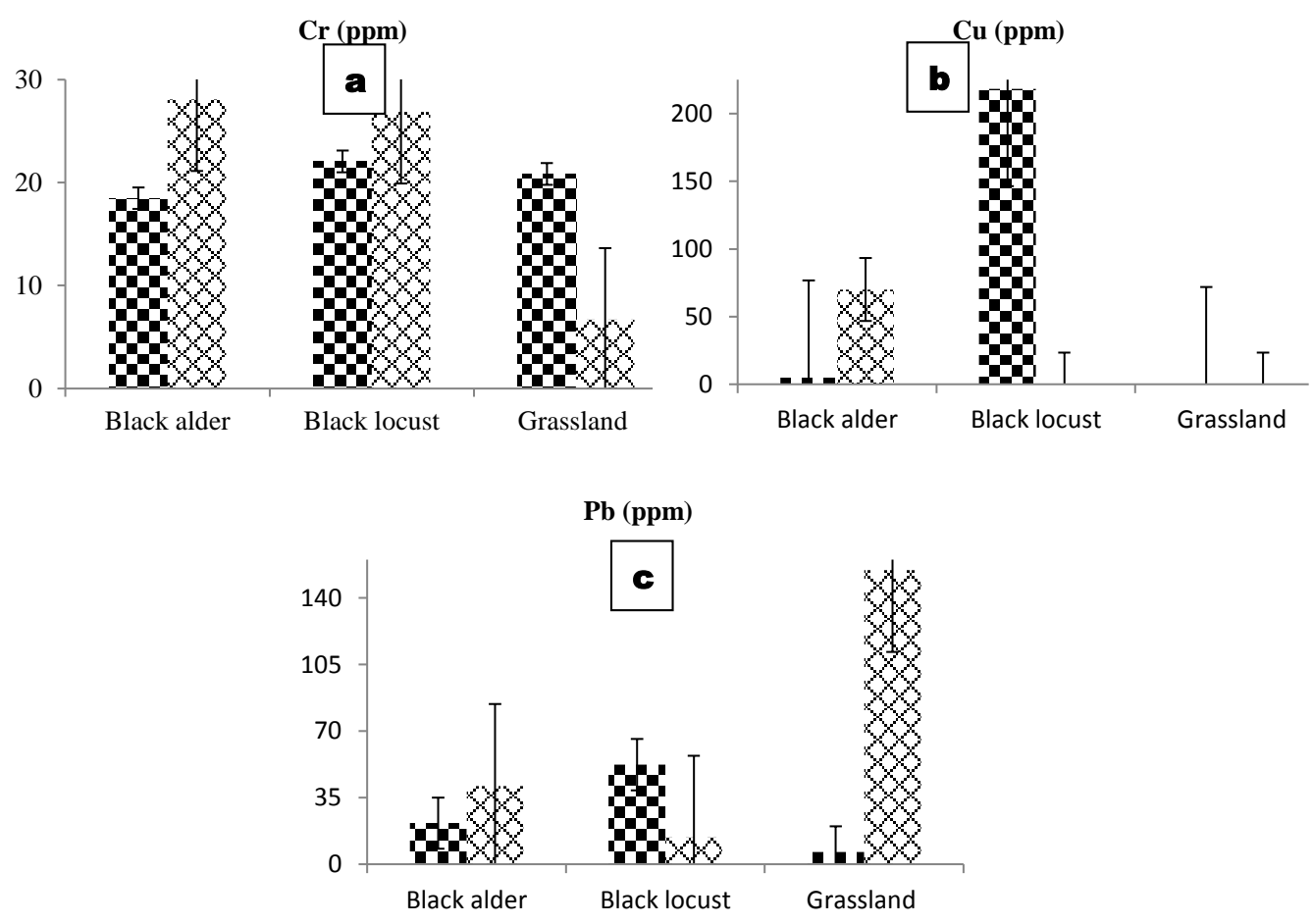

W North Facing Slopes

$\not x \times x$ South Facing Slopes

Figure 4. Heavy metal contents of soils significantly affected by LULC and slope aspect

$\mathrm{Cu}$ content of soils in the study area ranged from 0 to $380 \mathrm{ppm}$ with the average value of $49 \mathrm{ppm}$. This range is consistent with the range in the world ( 2 to $250 \mathrm{ppm}$ ) reported by Kabata-Pendias and Mukherjee (2007). Soil $\mathrm{Cu}$ concentration of black locust $(109.1 \mathrm{ppm})$ was significantly higher than that of grassland $(0.001 \mathrm{ppm})$ $(\mathrm{p}=0.007)$. While in north aspects, the lowest and the highest soil $\mathrm{Cu}$ concentrations belonged to grasslands ( $0.001 \mathrm{ppm})$ and black locust $(218.3 \mathrm{ppm})$, respectively, in south aspects, the lowest and highest soil $\mathrm{Cu}$ concentrations belonged to grassland $(0.001 \mathrm{ppm})$ and black alder respectively $(70.0 \mathrm{ppm})$ (Fig. 4b; Table 4). Higher $\mathrm{Cu}$ contents in forest soils might be related to higher clay contents than that in grassland soils $(\mathrm{r}=0.7 \mathrm{p}<0.001)$ (Table 2). Zheng et al. (2016) also reported positive correlation between $\mathrm{Cu}$ and clay contents of soil for coastal soils in China. 
$\mathrm{Pb}$ content of soils in the study area ranged from 0 to $254.2 \mathrm{ppm}$ with the average value of $48 \mathrm{ppm}$. This value is higher than that in the world (up to $90 \mathrm{ppm}$ ) KabataPendias and Mukherjee (2007). Pb concentration was found significantly highest in grassland soils (80.5 ppm) compared to black locust $(33.2 \mathrm{ppm})$ and black alder (31.3 ppm) soils. The $\mathrm{Pb}$ concentration significantly increased from north (26.8 ppm) to south $(69.9 \mathrm{ppm})(\mathrm{p}=0.002)$. In SFS, grassland soils had significantly higher $\mathrm{Pb}$ concentration than others $(\mathrm{p}<0.001)$. Grassland soil $\mathrm{Pb}$ concentration increased from north $(6.5 \mathrm{ppm})$ to SFS (154.5 ppm) (Fig. 4c; Table 4). The lower Pb content in forest soils may be related to higher $\mathrm{pH}(\mathrm{r}=-0.5 \mathrm{p}<0.01)$ and higher clay content $(\mathrm{r}=0.7$ $\mathrm{p}<0.001$ ) (Table 2). The lower Pb content in black alder and black locust may be related to phytoremediation (Escobar and Dussan, 2016; Babu et al., 2013; Escobar and Dussan, 2016; Lee et al., 2009). Alnus acuminata subsp. acuminata for example, took $\mathrm{Pb}$ and $\mathrm{Cr}$ up as $135 \mathrm{mg} / \mathrm{kg}$ and $71 \mathrm{mg} / \mathrm{kg}$, respectively (Escobar and Dussan, 2016).

Many studies have been performed to determine the effects of copper smelter on heavy metal concentration (Adamo et al., 2002; Dudka et al., 1996; Hutchinson and Whitby, 1977; Kuo et al., 1983). For example, Adamo et al. (2002) found the related heavy metal concentrations of soil near the smelter in Ontario, Canada as 43352.7 and $63 \mathrm{ppm}$ for $\mathrm{Cu}, \mathrm{Pb}, \mathrm{Zn}, \mathrm{Cd}$ and $\mathrm{Cr}$, respectively. Hutchinson and Whitby (1977) reported heavy metal concentrations in soils for $\mathrm{Cu}, \mathrm{Pb}$ and $\mathrm{Zn}$ near the Sudbury smelting region of Canada as 13451 and 62 ppm, respectively. Cai et al. (2015) reported the average heavy metal concentrations in soil near Tonglushan copper mine in Hubei, China for $\mathrm{Cu}, \mathrm{Pb}$ and $\mathrm{Cd}$ as 38120 and 2.59 ppm, respectively. While $\mathrm{Cu}, \mathrm{Pb}$, $\mathrm{Cd}$ and $\mathrm{Cr}$ concentrations in soils determined in the present study was consistent with those in the other studies (Adamo et al., 2002; Dudka et al., 1996; Hutchinson and Whitby, 1977; Kuo et al., 1983), Zn was found higher than the others.

\section{Comparison of main factors' effects with partial eta squared ( $\eta 2)$}

The anthropogenic index was computed using Equation 1. This index indicates which main factor was more effective on selected soil properties, natural (slope aspect) or anthropogenic (land use/land cover). This index was computed as 0.75 closer to indicates adverse human impact on soil properties.

The partial eta-squared values $(\eta 2)$ were also used to compare the effect size (explained proportion of variance by any factor) of main factors and their interaction on selected soil properties. The related values $(\eta 2)$ were presented in Tables 4 and Figure 5. Regarding soil texture, clay was the most affected texture component by land use, slope aspect and their interaction with $\eta 2$ values of 0.60 .59 and 0.1 respectively (Fig. 5). pH, EC and OM were the most significantly affected soil chemical properties after $\mathrm{Cr}$ by land use, slope aspect and their interaction respectively, were $\mathrm{pH}$ with values of 0.60 .10 .81 ; EC with values of 0.50 .30 .46 and $\mathrm{OM}$ with values of 0.20 .00 .31 (Fig. $5)$.

The most significantly affected soil properties by land use and interaction was found Cr with values of 0.93 and 0.94 (Fig. 5). The significant interaction effect on soil properties such as $\mathrm{pH}, \mathrm{OM}, \mathrm{TN}$, Stoniness and heavy metals except Cd was found higher than that of main factors. That means the interaction between main factors strengthen the effect. For example, while effect size of land use and slope aspect on $\mathrm{Pb}$ content were 0.29 and 0.27 respectively, the interaction effect was found 0.54 which was about two times higher than that of the other factors (Table 4). 
Table 4. Heavy metal contents (mean \pm standard error) of surface soils according to LULC and slope aspect

\begin{tabular}{|c|c|c|c|c|c|c|}
\hline LULC & SASP & Cd (ppm) & $\mathrm{Cr}$ (ppm) & $\mathrm{Cu}(\mathrm{ppm})$ & $\mathrm{Pb}(\mathrm{ppm})$ & $\mathrm{Zn}(\mathbf{p p m})$ \\
\hline \multirow{2}{*}{ Black locust } & NFS & $0.3 \pm 0.2$ a_A & $22.0 \pm 0.6$ a_A & 218.3 $\pm 66.0 \_$a_A & 52.3 \pm 17.6 _a_A & $140.6 \pm 8.0 \_$a_AB \\
\hline & SFS & $0.0 \pm 0.0$ a_A & $26.8 \pm 0.9$ b_A & $0.0 \pm 0.0 \_\mathrm{b} \_\mathrm{A}$ & $14.0 \pm 5.2 \_\mathrm{a} \_\mathrm{A}$ & 113.3 \pm 12.9 _a_A \\
\hline \multirow{2}{*}{ Black alder } & NFS & $0.7 \pm 0.3$-a_A & $18.5 \pm 0.5$ _a_B & 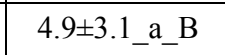 & 21.6 5 5.6_a_AB & 164.5 15.1 _a_B \\
\hline & SFS & $2.1 \pm 1.3 \_\mathrm{a} \_\mathrm{A}$ & $28.1 \pm 0.5 \_$b_A & 70.0 \pm 45.9 a_A & 41.1 \pm 22.7 a_A & $167.2 \pm 40.5 \_\mathrm{a} \_\mathrm{A}$ \\
\hline \multirow{2}{*}{ Grassland } & NFS & $0.0 \pm 0.0 \_\mathrm{a} \_\mathrm{A}$ & $20.8 \pm 0.4$ _a_A & $0.0 \pm 0.0 \_$a_B & $6.5 \pm 1.7$ _a_B & 119.5 11.8 _a_A \\
\hline & SFS & $1.0 \pm 0.3 \_\mathrm{a} \_\mathrm{A}$ & 6.7 $\pm 0.5 \_b \_B$ & $0.0 \pm 0.0 \_\mathrm{a} \_\mathrm{A}$ & 154.5 $\pm 25.1 \_b \_B$ & $186.0 \pm 12.1 \_\mathrm{a} \_\mathrm{A}$ \\
\hline \multirow{6}{*}{$\begin{array}{l}\text { Two-way } \\
\text { ANOVA }\end{array}$} & $\mathrm{IUJ} C$ & $\mathrm{~F}=2.4$ & $\mathrm{~F}=196.1$ & $\mathrm{~F}=5.7$ & $\mathrm{~F}=6.1$ & $\mathrm{~F}=2.0$ \\
\hline & LULC & $\mathrm{p}>0.05$ & $\mathrm{p}<0.001$ & $\mathrm{p}<0.01$ & $\mathrm{p}<0.01$ & $\mathrm{p}>0.05$ \\
\hline & SASP & $\mathrm{F}=2.2$ & $\mathrm{~F}=0.0$ & $\mathrm{~F}=3.6$ & $\mathrm{~F}=11.0$ & $\mathrm{~F}=0.0$ \\
\hline & SASP & $\mathrm{p}>0.05$ & $\mathrm{p}>0.05$ & $p>0.05$ & $\mathrm{p}<0.01$ & $\mathrm{p}>0.05$ \\
\hline & Int & $\mathrm{F}=1.2$ & $F=221.3$ & $\mathrm{~F}=10.2$ & $\mathrm{~F}=18.0$ & $\mathrm{~F}=2.9$ \\
\hline & mteraction & $\mathrm{p}>0.05$ & $\mathrm{p}<0.001$ & $\mathrm{p}<0.001$ & $\mathrm{p}<0.001$ & $\mathrm{p}>0.05$ \\
\hline
\end{tabular}

LULC: land-use/land cover, SASP: slope aspect. NFS: north facing slopes, SFS: south facing slopes. By slope aspect, values of same LULC follow the various lower-case letters indicate significant difference. By LULC, values of same slope aspect follow the various upper-case letters differ significantly

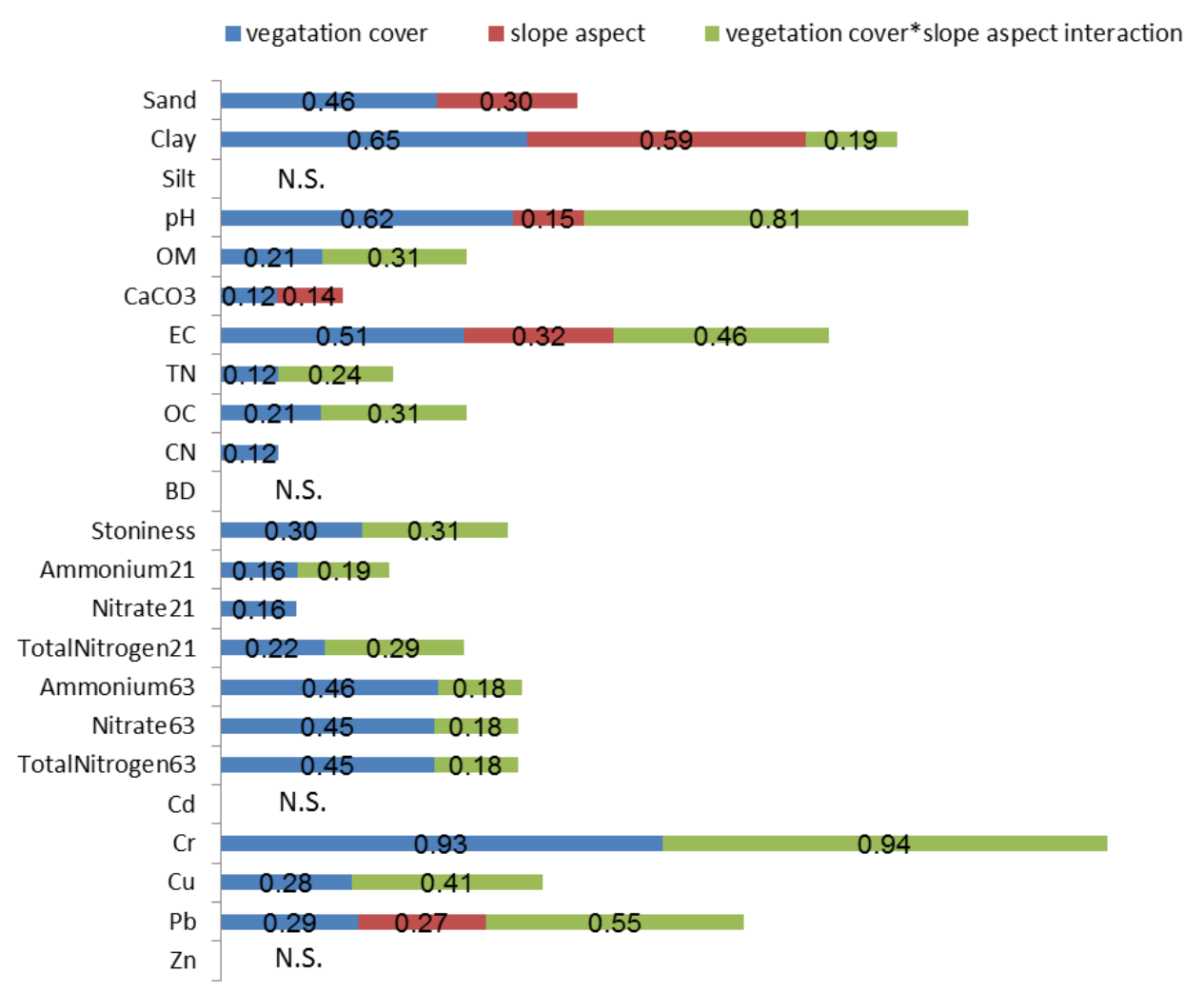

Figure 5. Effect size ( $\eta 2)$ of independent variables on selected soil properties

\section{Conclusion}

The results from the present study showed significant interaction effect beside LULC and slope aspect on selected soil properties. In general, main factors and their interaction improved selected soil properties affecting soil quality. The most affected 
soil properties by LULC and slope aspect were $\mathrm{Cr}$ content and clay content, respectively. The improving effects of afforestation compared to grasslands was revealed on only clay contents and soil skeleton among physical properties; $\mathrm{pH}$, nitrate and total nitrogen mineralization for 63-day among chemical properties. Our study showed that changes in LULC from grasslands to forest (especially to black alder) promoted soil quality in terms of total nitrogen and $\mathrm{NO}_{3}-\mathrm{N}$, playing a fundamental role in ecological restoration, beside other soil properties. Although decreasing effect (phytoremediation) of black alder and black locust on heavy metals except $\mathrm{Pb}$ in soil was not found In the present study, Alnus glutinosa and Robinia pseudoacacia may be suggested for the restoration/reclamation of mining soils in terms of phytoremediation beside their advantages such as improving nitrogen mineralization. However, further periodical studies are needed to determine the improving effects of afforestation on soil properties after mining. Results from the present study may be generalized to larger areas which are similar to the study area in terms of land-use and vegetation cover

Acknowledgements. This research was supported by Artvin Coruh University Scientific Research Project Unit, Turkey (Ref. No. 2016.F10.02.13). We thank Dr. Aydin Tufekcioglu and Dr. Funda OGUT from Artvin Coruh University for their valuable proofreading.

\section{REFERENCES}

[1] Acatay, A. (1968): Smoke damages from copper smelter factory in Murgul. - Journal of the Faculty of Forestry Istanbul University, Series A, 18(1): 1-17 (in Turkish).

[2] Adamo, P., Dudka, S., Wilson, M. J., McHardy, W. J. (2002): Distribution of trace elements in soils from the Sudbury smelting area (Ontario, Canada). - Water Air Soil Poll 137: 95-116.

[3] Allgaier, F. (1997): Environmental Effects of Mining. - In: Marcus, J. J. (ed.) Mining Environmental Handbook: Effects of Mining on the Environment and American Environmental Controls on Mining. World Scientific, River Edge, NJ, pp 132-189.

[4] Anonymous (2002): 1/500.000 Scaled Trabzon and Samsun Plates. - General Directorate of Mineral Research and Exploration, Ankara.

[5] Ansin, R. (1980): Flora of Eastern Black Sea Region and floristic content of primary vegetation types. - Associate Professorship Thesis, Karadeniz Technical University.

[6] Assefa, D., Rewald, B., Sanden, H., Rosinger, C., Abiyu, A., Yitaferu, B., Godbold, D. L. (2017): Deforestation and land use strongly effect soil organic carbon and nitrogen stock in Northwest Ethiopia. - Catena 153: 89-99. DOI: 10.1016/j.catena2017.02.003.

[7] Babu, A. G., Kim, J. D., Oh, B. T. (2013): Enhancement of heavy metal phytoremediation by Alnus firma with endophytic Bacillus thuringiensis GDB-1. - J Hazard Mater 250: 477-483.

[8] Bangroo, S., Najar, G., Rasool, A. (2017): Effect of altitude and aspect on soil organic carbon and nitrogen stocks in the Himalayan Mawer Forest Range. - Catena 158: 63-68.

[9] Beheshti, A., Raiesi, F., Golchin, A. (2012): Soil properties, C fractions and their dynamics in land use conversion from native forests to croplands in northern Iran. - Agr Ecosyst Environ 148: 121-133. DOI: 10.1016/j.agee.2011.12.001.

[10] Biro, K., Pradhan, B., Buchroithner, M., Makeschin, F. (2013): Land use/land cover change analysis and its impact on soil properties in the Northern Part of Gadarif region, Sudan. - Land Degrad Dev 24: 90-102. DOI: 10.1002/ldr.1116.

[11] Blake, G. R., Hartge, K. (1986): Bulk Density 1. - In: Klute, A. (ed.) Methods of Soil Analysis. Part 1: Physical and Mineralogical Methods. SSSA, ASA, Madison, WI, pp. 363-375. 
[12] Bouyoucos, G. J. (1962): Hydrometer method improved for making particle size analyses of soils. - Agronomy Journal 54: 464-465.

[13] Bremner, J., Keeney, D. R. (1965): Steam distillation methods for determination of ammonium, nitrate and nitrite. - Analytica Chimica Acta 32: 485-495.

[14] Cai, L. M., Xu, Z. C., Qi, J. Y., Feng, Z. Z., Xiang, T. S. (2015): Assessment of exposure to heavy metals and health risks among residents near Tonglushan mine in Hubei, China. - Chemosphere 127: 127-135.

[15] Chacon, G., Gagnon, D., Pare, D. (2009): Comparison of soil properties of native forests, Pinus patula plantations and adjacent pastures in the Andean highlands of southern Ecuador: land use history or recent vegetation effects? - Soil Use Manage 25: 427-433. DOI: 10.1111/j.1475-2743.2009.00233.x.

[16] Cui, J., Hirota, M., Kamijo, T., Yoshitake, S., Katoh, K. (2018): Soil net nitrogen mineralization at different ecosystem development stages after the year 2000 eruption on Miyakejima Island. - Journal of Ecosystem \& Ecography 8: 1-9. DOI: 10.4172/21577625.250 .

[17] Deng, X. Z., Zhao, C. H., Yan, H. M. (2013): Systematic modeling of impacts of land use and land cover changes on regional climate: a review. - Adv Meteorol. DOI: $10.1155 / 2013 / 317678$.

[18] DMI (2005): Meteorological Bulletin. - Turkish State Meteorological Service, Ankara.

[19] DPT (2001): Mining Special Commission Report. - DPT, Ankara.

[20] Dudka, S., PonceHernandez, R., Tate, G., Hutchinson, T. C. (1996): Forms of Cu, Ni, and $\mathrm{Zn}$ in soils of Sudbury, Ontario and the metal concentrations in plants. - Water Air Soil Poll 90: 531-542.

[21] EPA (1996): Microwave Assisted Acid Digestion of Sediments, Sludges, Soils, and Oils. OHW, Method 3051. - EPA, Washington.

[22] Erdin, K. (1983): Remote sensing in forestry and determination of smoke damages by infrared colour films (in Turkish). -

[23] Escobar, M. P., Dussan, J. (2016): Phytoremediation potential of chromium and lead by Alnus acuminata subsp acuminata. - Environ Prog Sustain 35: 942-948.

[24] Fischer, A., Fischer, H. (2006): Restoration of forests. In: van Andel, J., Aronson, J. (eds.). Restoration ecology. Blackwell Publishing, Malden, Massachusetts, U.S.A. p. 124-140.

[25] Gol, C. (2017): Effects of aspect and changes in land use on organic carbon and soil properties in Uludere catchment, semi-arid region: Turkey. - Rend Lincei-Sci Fis Nat 28: 463-469. DOI: 10.1007/s12210-017-0619-x.

[26] Guleryuz, G., Kirmizi, S., Arslan, H. (2007): Nitrogen mineralisation in the soils of alpine mat communities: an incubation experiment under laboratory conditions. - Turkish Journal of Botany 31: 277-286.

[27] Hart, S. C., Binkley, D., Perry, D. A. (1997): Influence of red alder on soil nitrogen transformations in two conifer forests of contrasting productivity. - Soil Biol Biochem 29: 1111-1123.

[28] Hazelton, P., Murphy, B. (2016): Interpreting Soil Test Results: What Do all the Numbers Mean? - CSIRO Publishing, Clayton.

[29] Hung, T. T., Doyle, R., Eyles, A., Mohammed, C. (2017): Comparison of soil properties under tropical Acacia hybrid plantation and shifting cultivation land use in northern Vietnam. - Southern Forests: A Journal of Forest Science 79(1): 9-18.

[30] Hutchinson, T., Whitby, L. (1977): The effects of acid rainfall and heavy metal particulates on a boreal forest ecosystem near the Sudbury smelting region of Canada. Water, Air, and Soil Pollution 7: 421-438.

[31] IBM.Corp (2011): IBM SPSS Statistics for Windows, Version 20.0. - IBM Corp, Armonk, NY.

[32] Johnson, K. D. et al. (2011): Soil carbon distribution in Alaska in relation to soil-forming factors. - Geoderma 167-68: 71-84. DOI: 10.1016/j.geoderma.2011.10.006. 
[33] Kabata-Pendias, A., Mukherjee, A. B. (2007): Trace Elements from Soil to Human. Springer Science \& Business Media, Berlin.

[34] Kalra, Y. P., Maynard, D. G. (1991): Methods Manual for Forest Soil and Plant Analysis (Vol. 319). - Forestry Canada, Northwest Region, Northern Forestry Centre, Edmonton, Alberta.

[35] Kucuk, M. (2013): Determination of nitrogen mineralization and soil respiration in different slope and aspect groups of oak stands and pasture. - Ph. D. Thesis, Karadeniz Technical University.

[36] Kuo, S., Heilman, P. E., Baker, A. S. (1983): Distribution and forms of copper, zinc, cadmium, iron, and manganese in soils near a copper smelter. - Soil Science 135: 101109.

[37] Lal, R. (2004): Soil carbon sequestration to mitigate climate change. - Geoderma 123:122.

[38] Lee, D.-B., Nam, W., Kwak, Y.-S., Cho, N.-H., Lee, S.-S. (2009): Phytoremediation of heavy-metal-contaminated soil in a reclaimed dredging area using Alnus species. Journal of Ecology and Environment 32: 267-275.

[39] Leech, N., Barrett, K., Morgan, G. A. (2013): SPSS for Intermediate Statistics: Use and Interpretation. - Routledge, New York.

[40] Liu, X. Y., Bai, Z. K., Zhou, W., Cao, Y. G., Zhang, G. J. (2017): Changes in soil properties in the soil profile after mining and reclamation in an opencast coal mine on the Loess Plateau, China. - Ecological Engineering 98: 228-239. DOI: 10.1016/j.ecoleng.2016.10.078.

[41] Mahmood, R. et al. (2010): Impacts of land use/land cover change on climate and future research priorities. - Bulletin of the American Meteorological Society 91: 37-46.

[42] Miheretu, B. A., Yimer, A. A. (2018): Spatial variability of selected soil properties in relation to land use and slope position in Gelana sub-watershed, Northern highlands of Ethiopia. - Phys Geogr 39: 230-245. DOI: 10.1080/02723646.2017.1380972.

[43] Negasa, T., Ketema, H., Legesse, A., Sisay, M., Temesgen, H. (2017): Variation in soil properties under different land use types managed by smallholder farmers along the toposequence in southern Ethiopia. - Geoderma 29: 40-50.

[44] Nelson, R. (1982): Carbonate and Gypsum. - In: Page, A. L. (ed.) Methods of Soil Analysis. Part 2: Chemical and Microbiological Properties. SSSA, ASA, Madison, WI, pp. 181-197.

[45] Oruc, N. (2013): Environmental effects of Murgul Copper Factory: a review. International Caucasia Forestry Symposium, Artvin Coruh University, October 24-26.

[46] Pansu, M., Gautheyrou, J. (2007): Handbook of soil Analysis: Mineralogical, Organic and Inorganic Methods. - Springer, Berlin Heidelberg.

[47] Pu, X., Cheng, H. G., Tysklind, M., Xie, J., Lu, L., Yang, S. T. (2018): Indications of soil properties on dissolved organic carbon variability following a successive land use conversion. $\quad-\quad$ Ecological Engineering 117: 115-119. DOI: 10.1016/j.ecoleng.2018.03.018.

[48] Qi, Y., Chen, T., Pu, J., Yang, F., Shukla, M. K., Chang, Q. (2018): Response of soil physical, chemical and microbial biomass properties to land use changes in fixed desertified land. - Catena 160: 339-344.

[49] Qin, Y.Y., Holden, N., Feng, Q., Zhu, M. (2017): Influence of Slope Aspect on Plant Community Composition and its Implications for Restoration of a Chinese Mountain Range. - Pol J Environ Stud 26: 375-383 doi:10.15244/pjoes/64458.

[50] Rezaei, S. A., Arzani, H., Tongway, D. (2006): Assessing rangeland capability in Iran using landscape function indices based on soil surface attributes. - Journal of Arid Environments 65: 460-473.

[51] Ripley, S.W., Krzic, M., Bradfield, G.E., Bomke, A.A. (2010): Land-use impacts on selected soil properties of the Yungas/Chaco transition forest of Jujuy province, 
northwestern Argentina: a preliminary study. - Canadian Journal of Soil Science 90: 679683 doi: $10.4141 /$ cjss09101.

[52] Shrestha, R. K., Lal, R. (2011): Changes in physical and chemical properties of soil after surface mining and reclamation. - Geoderma 161: 168-176.

[53] Tecimen, H. B., Sevgi, O., Yurtseven, H., Sevgi, E., Ozturk, M. (2013): Net nitrogen mineralization and nitrification rates in different land uses: an in situ incubation. - Fresen Environ Bull 22: 1173-1178.

[54] Tesfaye, M. A., Bravo, F., Ruiz-Peinado, R., Pando, V., Bravo-Oviedo, A. (2016): Impact of changes in land use, species and elevation on soil organic carbon and total nitrogen in Ethiopian Central Highlands. - Geoderma 261: 70-79. DOI: 10.1016/j.geoderma.2015.06.022.

[55] Thornthwaite, C. W. (1948): An approach toward a rational classification of climate. Geographical Review 38: 55-94.

[56] Toohey, R. C., Boll, J., Brooks, E. S., Jones, J. R. (2018): Effects of land use on soil properties and hydrological processes at the point, plot, and catchment scale in volcanic soils near Turrialba, Costa Rica. - Geoderma 315: 138-148. DOI: 10.1016/j.geoderma.2017.11.044.

[57] Tsehaye, G., Mohammed, A. A. (2013): Effects of land-use/cover changes on soil properties in a dryland watershed of Hirmi and its adjacent agro ecosystem: northern Ethiopia. - International Journal of Geosciences Research 1(1): 45-57.

[58] Tufekcioglu, A., Kucuk, M. (2004): Soil respiration in young and old oriental spruce stands and in adjacent grasslands in Artvin, Turkey. - Turkish Journal of Agriculture and Forestry 28: 429-434.

[59] TUIK (2013): Artvin with Selected Indicators. - Turkish Statistical Institute, Ankara.

[60] Uri, V., Lohmus, K., Kund, M., Tullus, H. (2008): The effect of land use type on net nitrogen mineralization on abandoned agricultural land: Silver birch stand versus grassland. - Forest Ecology and Management 255: 226-233.

[61] Wei, X. R., Shao, M. A., Fu, X. L., Agren, G. I., Yin, X. Q. (2011): The effects of land use on soil $\mathrm{N}$ mineralization during the growing season on the northern Loess Plateau of China. - Geoderma 160: 590-598.

[62] Wilson, B. R., Koen, T. B., Barnes, P., Ghosh, S., King, D. (2011): Soil carbon and related soil properties along a soil type and land-use intensity gradient, New South Wales, Australia. - Soil Use Manage 27: 437-447. DOI: 10.1111/j.1475-2743.2011.00357.x.

[63] Xu, X. L., Ma, K. M., Fu, B. J., Song, C. J., Liu, W. (2008): Relationships between vegetation and soil and topography in a dry warm river valley, SW China. - Catena 75 : 138-145.

[64] Xue, Z.J., Cheng, M., An, S.S. (2013): Soil nitrogen distributions for different land uses and landscape positions in a small watershed on Loess Plateau. - China Ecological Engineering 60: 204-213.

[65] Yener, I. (2013): Investigation of relationships between some ecological factors and the growth of pure oriental spruce (Picea orientalis (L.) link) forests at different sites. - Ph. D. Thesis, Karadeniz Technical University.

[66] Yesilonis, I., Szlavecz, K., Pouyat, R., Whigham, D., Xia, L. (2016): Historical land use and stand age effects on forest soil properties in the Mid-Atlantic US. - Forest Ecology and Management 370: 83-92. DOI: 10.1016/j.foreco.2016.03.046.

[67] Zhang, W. T., Weindorf, D. C., Zhu, Y. D., Haggard, B. J., Bakr, N. (2012): Soil series and land use impacts on major soil properties: a quantitative comparison. - Soil Res 50: 390-396. DOI: $10.1071 / \mathrm{sr} 11247$.

[68] Zheng, R., Zhao, J. L., Zhou, X., Ma, C., Wang, L., Gao, X. J. (2016): Land use effects on the distribution and speciation of heavy metals and arsenic in coastal soils on Chongming Island in the Yangtze River Estuary, China. - Pedosphere 26: 74-84. DOI: 10.1016/s1002-0160(15)60024-8. 